\title{
Metodologia para Definição de Instrumentos de Apoio a Iniciativas de Melhoria de Processos de Software Multi-Modelos Baseadas nos Modelos MR-MPS e CMMI-DEV
}

\author{
Marcelo Santos de Mello ${ }^{1,2}$, Ana Regina Rocha ${ }^{2}$, Gleison Santos ${ }^{3}$ \\ ${ }^{1}$ Informal Informática Ltda.
}

Rua do Catete, 311, Gr 1311 - CEP 22220-001 - Rio de Janeiro - RJ - Brasil

${ }^{2}$ Programa de Engenharia de Sistemas e Computação

COPPE/UFRJ - Universidade Federal do Rio de Janeiro

Caixa Postal 68511 - CEP 21945-970 - Rio de Janeiro, Brasil

${ }^{3}$ Programa de Pós-Graduação em Informática

Universidade Federal do Estado do Rio de Janeiro (UNIRIO)

Caixa Postal 56011- CEP 22290-970 - Rio de Janeiro, Brasil

marcelo@informal.com.br, darocha@cos.ufrj.br,gleison.santos@uniriotec.br

Resumo. A utilização de mais de uma norma de qualidade ou modelo de referência como guias para adoção de melhores práticas no desenvolvimento de software é cada vez mais comum pelas organizações de software que objetivam a melhoria de seus processos. Este artigo apresenta a metodologia utilizada para a definição de um mapeamento entre o CMMI-DEV e o MR-MPS de forma a auxiliar as organizações em iniciativas de melhoria de processos de software multi-modelos, seja no ambito das implementações ou das avaliações de processos. Ao ser utilizada, esta metodologia possibilitou a identificação das similaridades e diferenças entre os modelos e, de forma complementar, deu origem a instrumentos de apoio às iniciativas desta natureza.

\begin{abstract}
The adoption of more than one quality standard or reference model as a guide to software engineering best practices adoption is common in software organizations that aim to improve their processes. This paper presents the methodology used to define a mapping between the CMMI-DEV and MR-MPS in order to support organizations in conduct multi-model software processes improvement initiatives. When used, this methodology enabled the identification of similarities and differences between the models and, in a complementary manner, has resulted in tools to support such initiatives.
\end{abstract}

\section{Introdução}

Para apoiar a implementação da melhoria em processos de software, normas e modelos de referência têm sido desenvolvidos e aprimorados com o objetivo de estabelecer melhores práticas na definição dos processos, e para servir de apoio à avaliação da capacidade e maturidade das organizações na produção de software (RESENDE et al., 2009).

Nos últimos anos, apesar do crescimento geral da adoção de normas e modelos de referência para melhoria de processos, a quantidade de organizações que adotam esses modelos ainda é uma parcela reduzida da população total das organizações de software (STAPLES et al., 2007). Embora existam uma variedade de modelos de refe- 
rência de processo amplamente aceitos, apenas um número reduzido destas organizações consegue sistematizar com sucesso seu processo de software em concordância com os modelos disponíveis (THIRY et al., 2008a).

Por outro lado, a definição do escopo de uma iniciativa de melhoria de processos de software vai além da escolha de um determinado modelo de referência, exigindo em muitos casos combinar modelos entre si e com os objetivos particulares de cada organização (ARAÚJO et al., 2004). Para que a utilização de mais de uma norma ou modelo pelas organizações produza os resultados esperados, sem redundância de definições e impacto no planejamento da melhoria, principalmente em relação ao tempo, custo e esforço, é importante avançar no sentido de identificar interseções e sobreposições das normas de qualidade e dos modelos de referência de processo (SOUZA et al., 2009).

Este trabalho apresenta a metodologia utilizada para a definição do mapeamento entre os modelos MR-MPS (SOFTEX, 2009a) e CMMI-DEV (SEI, 2006a), de forma a auxiliar as organizações em iniciativas de melhoria de processos de software multimodelos, seja no âmbito das implementações ou das avaliações de processos. O resultado desse mapeamento, parte de uma dissertação de mestrado (MELLO, 2011) deu origem ao Guia de Implementação do MR-MPS Parte 11 (SOFTEX, 2011), o qual possui orientações para a implementação e avaliação do MPS em conjunto com o CMMI-DEV.

O artigo está organizado em 6 seções, incluindo esta introdução. A Seção 2 apresenta iniciativas de melhoria de processos de software multi-modelos em organizações e alguns trabalhos realizados para mapeamento, integração e harmonização de normas e modelos de referência. As Seções 3 e 4 apresentam, respectivamente, a metodologia de pesquisa e a estrutura geral do mapeamento. A Seção 5 descreve a avaliação do mapeamento na indústria através de estudos de caso, relatando os principais resultados, enquanto que, por fim, a Seção 6 apresenta as considerações finais desse trabalho.

\section{Iniciativas e Trabalhos sobre Melhoria de Processos de Software Multi- Modelos}

A utilização de mais de uma norma de qualidade ou modelo de referência como guias para adoção de melhores práticas no desenvolvimento de soluções é cada vez mais comum no contexto de organizações de software que objetivam a melhoria de seus processos. A partir da revisão da literatura realizada no contexto da pesquisa, alguns relatos de iniciativas multi-modelos em organizações puderam ser observados.

NUNES et al. (2005) relatam uma experiência de melhoria de processos contemplando a norma ISO 9001 (ISO, 2008) e o modelo CMMI-DEV para alcance do nível 2 de maturidade concomitantemente, observando que um processo de software aderente a um determinado nível do CMMI-DEV pode ser também aderente à ISO 9001, no que se refere à Engenharia de Software. A partir do objetivo estabelecido de ampliar a participação no mercado e atender à crescente exigência dos clientes de produtos com qualidade assegurada, o projeto foi definido e organizado em 10 fases, da definição da coordenação do projeto até a avaliação do CMMI (Readiness Assessment).

Em FERREIRA et al. (2006) são apresentados resultados quantitativos do programa de melhoria de processos de uma organização que adotou a norma ISO 9001 e os modelos MPS e CMMI e que alcançou benefícios, envolvendo redução do retrabalho, diminuição de custos, aumento da motivação e melhoria de produtividade da equipe, com aumento da satisfação dos seus clientes. 
Em MELLO et al. (2009) é relatada uma experiência de implementação do MRMPS em conjunto com a ISO 9001, realizado em dois ciclos consecutivos de melhoria, com apresentação do mapeamento definido entre os modelos, em nível do item da norma para o processo do modelo, dificuldades encontradas e fatores de sucesso do projeto. Dentre as características apresentadas neste relato, podem ser citadas: implantação do Sistema de Gestão da Qualidade organizado segundo os itens da norma ISO 9001; aproveitamento da mesma documentação, produtos de trabalho e evidências para avaliação e auditoria; produção de artefatos de projeto de forma independente, mas com referência no Sistema de Gestão da Qualidade; realização de reuniões de acompanhamento do projeto de melhoria no contexto da análise crítica da ISO; utilização de ferramentas comuns; e definição de um processo único de mudança para evolução dos processos.

Um ponto importante a ser considerado na adoção de mais de um modelo pelas organizações está relacionado à documentação produzida. THIRY et al. (2008b) descrevem que, no contexto das avaliações integradas envolvendo mais de um modelo de referência, há um esforço com a gerência de documentos, tanto na fase preparatória como na documentação dos resultados, o que pode acarretar redundância.

Aprovadas no ano de 2009 pela coordenação do programa MPS.BR, as avaliações conjuntas MPS/CMMI-DEV começaram a ser adotadas pelas organizações para, entre outros aspectos, otimizar o tempo e o esforço do processo. Das lições aprendidas relatadas em SOUZA et al. (2009) após a primeira avaliação conjunta dos níveis de maturidade C do MPS e 3 do CMMI-DEV, observou-se que as diferenças de exigência entre os dois modelos ocasionaram a produção de resultados diferentes para as caracterizações dos processos da organização em ambos os modelos.

Outro importante aspecto destacado está relacionado ao entendimento prévio, por parte dos avaliadores e representantes da empresa na equipe de avaliação, das sutis diferenças e compatibilidades entre os modelos, de forma a evitar que, de fato, fosse realizada uma avaliação dupla ao invés de uma avaliação conjunta. Apesar de o MRMPS ser considerado compatível com o CMMI-DEV, e isso ter guiado a definição o modelo desde o seu princípio, não havia um documento explicitando as diferenças e similaridades entre ambos.

Ainda a partir dos relatos, observamos que o mapeamento entre modelos de referência também traz benefícios para a evolução dos próprios modelos. BECKER et al., (2007) apresentam um conjunto de oportunidades de melhoria para os níveis iniciais do modelo MPS, sugerindo alternativas de evolução elaboradas por integrantes do grupo de estudos, implementadores e empresas participantes do projeto Cooperativa MPS.BR SOFTSUL. Nesse relato, concluiu-se que iniciativas de organização de grupos de estudos, similares à citada no artigo, podem contribuir significativamente para o aperfeiçoamento e consolidação de modelos de melhoria de processos, pois os grupos podem proporcionar um foro de aprendizado coletivo, trocas de experiências, aquisição e disseminação de conhecimento.

Alinhados a esse contexto, algumas normas e modelos de referência indicam o que deve ser feito e outras descrevem como fazê-lo. Nas iniciativas de melhoria de processo, os diversos modelos de referência podem auxiliar as organizações, cada um alavancando suas melhores práticas. Entretanto, isso pode acarretar em custo e esforço adicionais, aumentando o risco de ineficiências e redundâncias.

Assim, pode ser importante harmonizar os frameworks de qualidade, ou seja, 
identificar intersecções e sobreposições, criando soluções de melhoria de processo multi-modelos. BALDASSARRE et al. (2010) relatam este entendimento e, a partir dele, propõem um processo de harmonização de apoio às organizações, abrangendo a combinação entre a ISO 9001 e o CMMI-DEV e mostrando como o GQM (BASILI e ROMBACH, 1988) pode ser utilizado para definir objetivos operacionais que atendam aos requisitos da ISO 9001 de forma reutilizável nas avaliações CMMI (Figura 1).

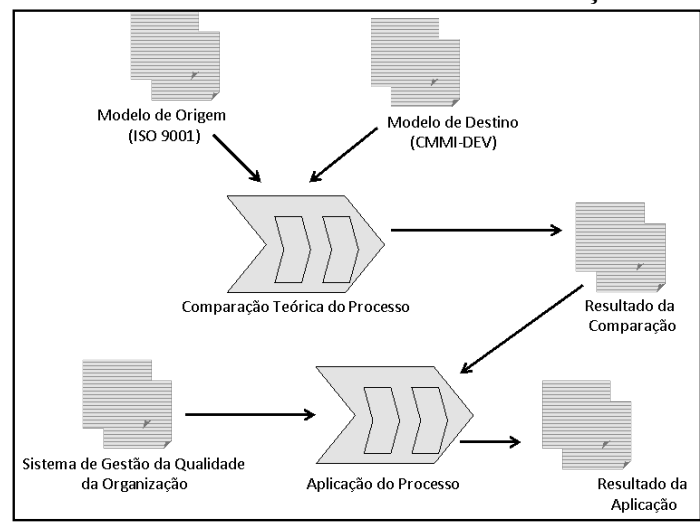

Figura 1 - Processo de Harmonização (BALDASSARRE et al., 2010)

Conduzida pelo grupo denominado PrIME (Process Improvement in Multimodel Environments), um método de harmonização foi proposto no contexto de uma pesquisa desenvolvida pelo SEI (Software Engineering Institute), onde foi definida uma abordagem para as iniciativas de melhoria multi-modelos, tendo como objetivo a integração de diferentes modelos, e contendo as seguintes principais etapas: alinhamento entre os objetivos organizacionais e de melhoria, identificação de tecnologias ou modelos de referência candidatos, categorização estratégica das tecnologias e o projeto e a implementação da solução de melhoria (KIRWAN et al., 2008b).

Nos projetos de melhoria multi-modelos, a integração dos modelos é uma preocupação constante das organizações. Entretanto, o agrupamento dos padrões não é um caminho estritamente preciso. A sobreposição entre eles é inevitável, pois a concepção dos modelos de referência possui desenvolvimento, tempo e pessoas envolvidas diferentes (MUTAFELIJA et al., 2009).

Neste sentido, cada modelo possui um conjunto específico de material de treinamento, guias e abordagem para avaliação, além de um conjunto distinto de termos e linguagens. Em seu trabalho de construção de um modelo integrado, MUTAFELIJA et al. (2009) orientam a exploração de similaridades e diferenças entre os padrões para capitalizar a sinergia, debatendo como as evidências necessárias para medir a conformidade com múltiplos padrões podem ser um caminho eficiente.

\section{Metodologia da Pesquisa}

Considerando as principais dificuldades e os problemas da adoção de mais de uma norma ou modelo de referência pelas organizações, observou-se a importância de identificar as interseções e sobreposições entre os modelos MPS e CMMI-DEV, bem como a relevância de elaborar instrumentos de apoio para realização de avaliações conjuntas de processos envolvendo os dois modelos. Para tal, um mapeamento foi conduzido com o objetivo de auxiliar as organizações nas iniciativas de melhoria de processos de software multi-modelos, seja no âmbito das implementações ou das avaliações de processos. Este mapeamento foi orientado por uma metodologia que possibilitou identificar as si- 
milaridades e diferenças entre os modelos e, de forma complementar, produzir instrumentos de apoio às iniciativas desta natureza.

A metodologia adotada foi estruturada nas etapas de revisão da literatura, elaboração do mapeamento e avaliação do mapeamento por revisão por pares e de estudos de caso na indústria, apresentadas a seguir. Um esquema da metodologia adotada pode ser visto na Figura 2. Os estudos de caso foram conduzidos de forma não concomitante.

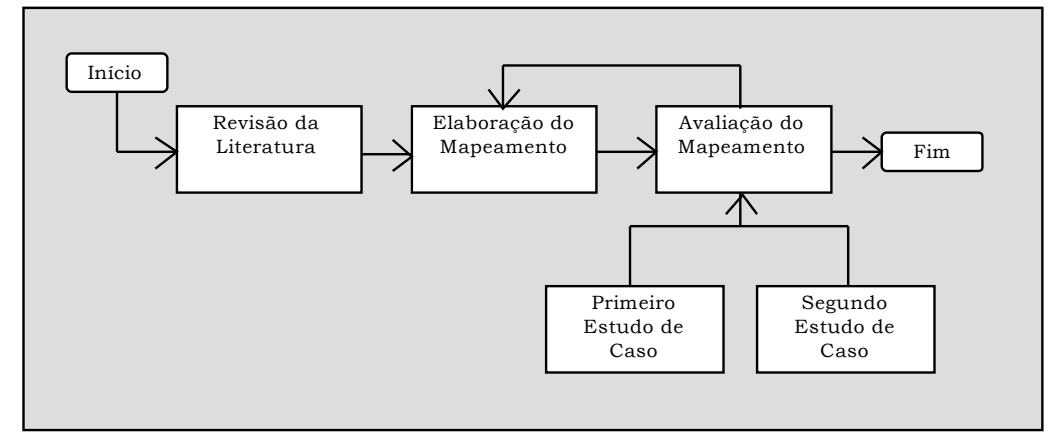

Figura 2 - Visão Geral da Metodologia de Pesquisa Adotada

\subsection{Revisão da Literatura}

No contexto do trabalho, a etapa de revisão da literatura teve como objetivo identificar, analisar e selecionar os estudos disponíveis relacionados ao assunto, no sentido de ampliar a compreensão das iniciativas de melhoria de processo de software multi-modelos.

Além da revisão informal, um estudo baseado em revisão sistemática (KITCHENHAM, 2008) foi planejado. O objetivo do estudo foi analisar relatos de experiência e publicações científicas sobre utilização de normas ISO e dos modelos MPS e CMMI-DEV, com o propósito de identificar abordagens, métodos e processos com relação ao mapeamento das normas e modelos, do ponto de vista dos pesquisadores e no contexto acadêmico e industrial. Foram identificadas 10 publicações. Os resultados completos obtidos podem ser vistos em detalhes em (MELLO, 2011). Em geral, percebeu-se que, com as informações extraídas, foi possível responder parcialmente às questões de pesquisa definidas para este estudo de revisão sistemática.

Com relação à questão principal de pesquisa ("Que abordagens, técnicas e processos têm sido propostos e/ou utilizados para mapeamento, integração e harmonização dos modelos MPS, CMMI-DEV e/ou normas ISO?"), de forma geral, há processos que buscam fornecer mecanismos de harmonização dos modelos, com métodos bem definidos e comparação em níveis mais baixos de abstração, permitindo maior precisão de análise (BALDASSARRE et al., 2010); outros buscam a geração de modelos integrados, produzidos a partir do mapeamento dos modelos e estruturados em sua maioria pela norma ISO 9001, por ter requisitos mais genéricos do que os modelos de referência de processos de software (CHANWOO et al., 2004). No entanto, não foram encontrados trabalhos relacionados ao mapeamento do modelo MPS com outras normas e modelos.

Para a primeira questão secundária de pesquisa ("Quais os critérios têm sido propostos e/ou adotados para mapeamento, integração e harmonização dos modelos MPS, CMMI-DEV e/ou normas ISO?'), verificou-se o uso da linguagem UML para representação dos conceitos e relação dos modelos de forma comum (LEPASAAR et al., 2002); de critérios mais específicos relacionados ao atendimento de requisitos de um modelo, em comparação a requisitos de outro modelo (CHANWOO et al., 2004). Tam- 
bém foi verificada a adoção de critérios associados a atributos específicos criados para a análise e posterior comparação dos modelos, envolvendo tamanho e complexidade, respectivamente baseados no escopo e estrutura de cada modelo (FERREIRA et al., 2010).

Outro aspecto observado foi a utilização de escalas de comparação, obtidas a partir de valores numéricos, indicando graus de relacionamento entre os modelos e respectivos componentes, a saber: $\mathrm{S}$ - fortemente relacionada $(86 \%$ a $100 \%)$; $\mathrm{L}$ - largamente relacionada $(51 \%$ a $85 \%) ; \mathrm{P}$ - parcialmente relacionada $(16 \%$ a $50 \%)$; W - fracamente relacionada (1\% a 15\%); Não relacionada (0\%) (PINO et al., 2010).

Para a terceira questão secundária ("Quais são as características das iniciativas de melhoria de processos de software multi-modelos em organizações?"), poucos relatos foram encontrados. Houve uma complementação pela revisão informal, que agregou trabalhos e estudos citados por diversos autores dos artigos selecionados, assim como experiências mais recentes ainda não disponíveis nas fontes definidas neste estudo.

Com esse estudo foram obtidas informações relevantes que permitiram: (a) identificar a existência de abordagens, métodos e processos adotados para mapeamento, integração e harmonização de normas e modelos de referência de processos; (b) identificar critérios de comparação entre estes modelos; e (c) identificar experiências de iniciativas de melhoria de processos de software multi-modelos em organizações.

\subsection{Elaboração do Mapeamento dos Modelos MPS e CMMI-DEV}

Para revelar as similaridades e diferenças entre as normas e modelos de referência de processo, o mapeamento é uma das estratégias mais observadas na literatura. Mapeamentos desta natureza, quando elaborados, devem ser claros, completos e não ambíguos (ROUT E TUFFLEY, 2007). Considerando o cenário das organizações brasileiras, os modelos MPS e CMMI-DEV, por serem os mais utilizados no apoio a iniciativas de melhoria de processos de software, foram selecionados para o mapeamento, abrangendo os processos e áreas de processos, respectivamente, para todos os níveis de maturidade. Foi realizado o mapeamento do MR-MPS com o CMMI-DEV (e não também na direção oposta) devido ao fato de as diferenças e similaridades poderem ser identificadas apenas com a execução desse mapeamento. Além disso, devido ao formato de representação escolhido, o mapeamento inverso pode ser derivado caso necessário.

De uma forma geral, as etapas para realização do mapeamento foram definidas a partir dos trabalhos consultados, em especial o de BALDASSARRE et al. (2010), tendo um modelo como origem e outro como destino. Composta por cinco atividades, esta etapa da metodologia é composta por (Figura 3): análise dos componentes dos modelos, definição dos critérios de classificação, definição do formulário padrão, comparação dos processos e avaliação através de revisão por pares. Todas são descritas na Seção 4.

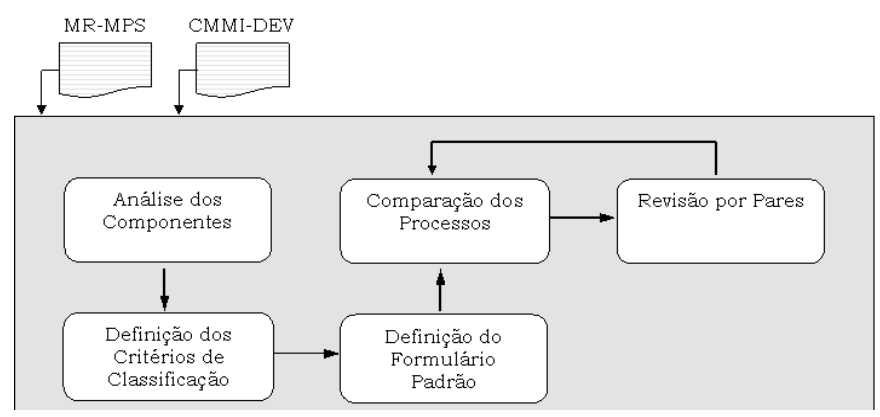

Figura 3 - Estrutura para Elaboração do Mapeamento 


\subsection{Avaliação do Mapeamento na Indústria}

Para avaliar o mapeamento foram seguidos os princípios da Engenharia de Software Experimental, na qual é possível obter a caracterização de determinada tecnologia em uso, sendo possível determinar com níveis razoáveis de segurança o que funciona e o que não funciona sob quais circunstâncias (MAFRA e TRAVASSOS, 2006).

Assim, após os ajustes no mapeamento realizados na etapa anterior, em resultado da revisão por pares, nesta etapa da metodologia o mapeamento foi avaliado através de dois estudos de caso, conduzidos com o objetivo de avaliar, em ambiente industrial, a corretude, a adequação dos critérios de comparação, o nível de detalhe, a utilidade das considerações e o desempenho dos avaliadores em uma avaliação conjunta.

O planejamento e a execução deste estudo experimental utilizou os conceitos do processo de experimentação definidos por WÖHLIN et al. (2000), composto pelas seguintes atividades: definição e planejamento; execução do estudo; análise de resultados; e empacotamento do estudo. O detalhamento do estudo pode ser visto na Seção 5.

\section{Mapeamento dos Modelos MPS e CMMI-DEV}

Para realização do mapeamento foi seguida a metodologia de pesquisa descrita na seção anterior, especificamente na etapa "Elaboração do Mapeamento", a qual é realizada através de cinco atividades, descritas nas próximas seções: análise dos componentes, definição dos critérios de classificação, definição do formulário padrão, comparação dos processos e avaliação através de revisão por pares.

\subsection{Análise dos Componentes dos Modelos}

A primeira atividade para elaboração do mapeamento teve como objetivo obter um entendimento da estrutura dos dois modelos e onde cada modelo descreve os seus componentes requeridos, isto é, obrigatórios, foco da comparação. Esta análise foi realizada após um estudo detalhado do MR-MPS:2009 (SOFTEX, 2009a) e do CMMI-DEV versão 1.2 (SEI, 2006a).

O MR-MPS define níveis de maturidade que são uma combinação de processos e atributos de processo. Processos estão descritos através de seu propósito e resultados esperados do processo, enquanto que os atributos de processo estão descritos através de resultados de atributos de processos (RAP). Neste sentido, os componentes requeridos no MR-MPS são os resultados esperados de processos e os resultados de atributos de processos. Já o CMMI-DEV está organizado em áreas de processos, com objetivos e práticas específicos e em objetivos e práticas genéricos. No CMMI-DEV, os componentes são agrupados em 3 categorias: requeridos, esperados e informativos. Em uma avaliação, os componentes requeridos e esperados do modelo CMMI-DEV têm o mesmo impacto dos resultados esperados do processo e dos resultados de atributos de processos do MR-MPS, que obrigatoriamente devem estar atendidos pela organização avaliada.

Desta forma, como resultado da análise dos componentes dos dois modelos, foram considerados para o mapeamento: (i) os processos do MR-MPS:2009 (SOFTEX, 2009a), comparados com as áreas de processo do CMMI-DEV versão 1.2 (SEI, 2006a); (ii) os resultados esperados dos processos do MR-MPS, comparados com as práticas específicas das áreas de processo do CMMI-DEV; (iii) os resultados de atributos de processos do MR-MPS, comparados com as práticas genéricas do CMMI-DEV; e (iv) nos níveis A e B do modelo MPS e 4 e 5 do modelo CMMI-DEV, os resultados de atributos de processos destes níveis são comparados também com as áreas de processos 
dos níveis 4 e 5 e suas respectivas práticas específicas.

Os objetivos específicos e genéricos do modelo CMMI-DEV foram excluídos da comparação porque eles não são avaliados diretamente, mas sim, através das práticas específicas e genéricas. Os objetivos do CMMI-DEV não têm correspondentes na estrutura do modelo MPS. Os componentes informativos do modelo CMMI-DEV, dentre eles as subpráticas e os produtos de trabalho típicos, foram excluídos da comparação, da mesma forma que as orientações para implementação dos resultados esperados do MRMPS, descritas nos guias de implementação (SOFTEX, 2009a). Em ambos os casos, tratam-se apenas de orientações e esclarecimentos adicionais, não sendo itens requeridos dos modelos.

No caso das práticas específicas e genéricas do CMMI-DEV, apenas sua declaração, segundo a tradução oficial para o português do modelo (SEI, 2006c), foi considerada no mapeamento, de forma que a descrição na forma de alternativas aceitáveis, também denominadas práticas alternativas, apesar de previstas no modelo, não foram mapeadas, pois são definidas para cada situação e organização específicas.

\subsection{Definição dos Critérios de Classificação}

A partir da revisão da literatura, etapa 1 da metodologia, foi identificada a importância da definição de critérios claros de comparação entre os modelos. Neste sentido, as seguintes categorias de classificação foram criadas: (i) Equivalente (EQU): As exigências do MR-MPS são exatamente as mesmas exigências do CMMI-DEV; (ii) Equivalente em conjunto $(E Q U+)$ : As exigências do MR-MPS são exatamente as mesmas exigências do CMMI-DEV quando complementadas com mais de um resultado esperado ou prática ou vice-versa; (iii) Não equivalente (NEQ): As exigências do MR-MPS não são exatamente as mesmas exigências do CMMI-DEV ou vice-versa; e (iv) Inexistente (INE): Não existe o resultado do MR-MPS no CMMI-DEV ou vice-versa.

Com os critérios de classificação definidos, foram estabelecidos roteiros de formulário para apoiar a comparação entre os processos e áreas de processos dos modelos, o que será mostrado na próxima seção.

\subsection{Definição do Formulário Padrão}

Quatro modelos de formulário padrão foram definidos para apoiar o trabalho de mapeamento, sempre considerando o MPS como modelo de origem e o CMMI-DEV como modelo de destino. O primeiro modelo de formulário (Figura 4) foi definido para ser utilizado no mapeamento de todos os processos e áreas de processo, com exceção do processo Gerência de Projetos do modelo MPS, por este estar relacionado com mais de uma área de processo do CMMI-DEV.

\begin{tabular}{|c|c|c|c|c|c|}
\hline \multirow{2}{*}{\multicolumn{2}{|c|}{ Resultado Esperado do Processo }} & \multicolumn{2}{|c|}{ Objetivo e Prática Específica } & \multicolumn{2}{|c|}{ Classificação e Considerações } \\
\hline & & $\begin{array}{l}\text { <prefixo e número do } \\
\text { objetivo específico }>\end{array}$ & $<$ declaras & & \\
\hline \begin{tabular}{|c|} 
sigla do resulta- \\
do esperado do \\
processo $>$
\end{tabular} & \begin{tabular}{|c|}
$<$ texto do resultado \\
esperado do pro- \\
cesso $>$
\end{tabular} & $\begin{array}{l}<\text { prefixo e número da } \\
\text { prática específica }>\end{array}$ & $\begin{array}{c}<\text { declaração da } \\
\text { prática específica }>\end{array}$ & $\begin{array}{c}<\text { classifica- } \\
\text { ção }>\end{array}$ & $\begin{array}{c}<\text { considera- } \\
\text { ções }>\end{array}$ \\
\hline
\end{tabular}

Figura 4 - Primeiro modelo de formulário

O segundo modelo de formulário (Figura 5) foi definido para ser utilizado no mapeamento dos resultados esperados do processo de Gerência de Projetos do MR-MPS com as práticas específicas das áreas de processo correspondentes do CMMI-DEV. Este processo teve um formulário específico pois o processo do MR-MPS é equivalente a 3 áreas de processo do CMMI-DEV. 


\begin{tabular}{|c|c|c|c|c|c|}
\hline \multicolumn{2}{|c|}{ Resultado Esperado do Processo } & \multicolumn{2}{|c|}{ Área de Processo e Prática Específica } & \multicolumn{2}{|c|}{ Classificação e Considerações } \\
\hline $\begin{array}{c}<\text { sigla do resultado } \\
\text { esperado do proces- } \\
\text { so }>\end{array}$ & $\begin{array}{c}<\text { texto do resul- } \\
\text { tado esperado } \\
\text { do processo }>\end{array}$ & $\begin{array}{l}<\text { área de processo e } \\
\text { prefixo e número da } \\
\text { prática específica }>\end{array}$ & $\begin{array}{c}<\text { declaração da } \\
\text { prática específi- } \\
\text { ca }>\end{array}$ & $\begin{array}{l}<\text { classifica- } \\
\quad \text { ção }>\end{array}$ & $\begin{array}{c}<\text { considera- } \\
\text { ções }>\end{array}$ \\
\hline
\end{tabular}

Figura 5 - Segundo modelo de formulário

O terceiro modelo formulário (Figura 6) foi definido para ser utilizado no mapeamento dos atributos de processo (AP) e resultados de atributo de processo (RAP) com os objetivos e práticas genéricas do CMMI-DEV até o AP 2.2, pois a partir deste ponto há correspondência entre RAP e práticas específicas de uma área de processo.

\begin{tabular}{|c|c|c|c|c|c|}
\hline $\begin{array}{c}\text { Atributo de Processo e Resultado de } \\
\text { Atributo de Processo }\end{array}$ & Objetivo e Prática Genérica & \multicolumn{2}{|c|}{ Classificação e Considerações } \\
\hline $\begin{array}{c}<\text { sigla do atributo de } \\
\text { processo }>\end{array}$ & $\begin{array}{c}\text { texto do atributo } \\
\text { de processo }>\end{array}$ & $\begin{array}{c}<\text { prefixo e número do } \\
\text { objetivo genérico }>\end{array}$ & \multicolumn{2}{|c|}{$<$ declaração do objetivo genérico $>$} \\
\hline $\begin{array}{c}<\text { sigla do resultado } \\
\text { de atributo de pro- } \\
\text { cesso }>\end{array}$ & $\begin{array}{c}\text { <texto do resulta- } \\
\text { do de atributo de } \\
\text { processo }>\end{array}$ & $\begin{array}{c}<\text { prefixo e número da } \\
\text { prática genérica }>\end{array}$ & $\begin{array}{c}<\text { declaração da } \\
\text { prática genérica }>\end{array}$ & $\begin{array}{c}<\text { classifica- } \\
\text { ção }>\end{array}$ & $\begin{array}{c}<\text { considera- } \\
\text { ções }>\end{array}$ \\
\hline
\end{tabular}

Figura 6 - Terceiro modelo de formulário

O quarto e último modelo de formulário (Figura 7) foi definido para ser utilizado no mapeamento dos atributos de processo (AP) e resultados de atributo de processo (RAP) a partir do AP 3.1, de forma a refletir a correspondência entre os AP e RAP e os objetivos e práticas genéricas, bem como com as respectivas práticas específicas das áreas de processo do CMMI-DEV.

\begin{tabular}{|c|c|c|c|c|c|c|}
\hline \multicolumn{2}{|c|}{$\begin{array}{l}\text { Atributo de Processo e Resultado de } \\
\text { Atributo de Processo }\end{array}$} & \multicolumn{2}{|c|}{$\begin{array}{l}\text { Objetivo e Prática Genérica e Área de } \\
\text { Processo e Prática Específica }\end{array}$} & \multicolumn{3}{|c|}{ Classificação e Considerações } \\
\hline $\begin{array}{c}<\text { sigla do atributo de } \\
\text { processo }>\end{array}$ & $\begin{array}{c}<\text { texto do atributo } \\
\text { de processo }>\end{array}$ & \begin{tabular}{|l}
$<$ prefixo e número do \\
objetivo genérico $>$
\end{tabular} & \multicolumn{4}{|c|}{ < declaração do objetivo genérico > } \\
\hline $\begin{array}{c}<\text { sigla do resultado } \\
\text { de atributo de pro- } \\
\text { cesso }>\end{array}$ & $\begin{array}{c}<\text { texto do resulta- } \\
\text { do de atributo de } \\
\text { processo }>\end{array}$ & $\begin{array}{c}<\text { prefixo e número da } \\
\text { prática genérica }>\text { ou } \\
<\text { área de processo e } \\
\text { prefixo e número da } \\
\text { prática especifica }>\end{array}$ & $\begin{array}{r}<\text { declaração da } p \\
\text { ca genérica }>o \\
<\text { declaração da } p \\
\text { ca específica }>\end{array}$ & & $<$ classificação $>$ & $\begin{array}{l}<\text { conside- } \\
\text { rações }>\end{array}$ \\
\hline
\end{tabular}

Figura 7 - Quarto modelo de formulário

Como o modelo de origem foi definido como sendo o MR-MPS, todos os formulários foram inicialmente preenchidos com as informações do modelo MPS.

\subsection{Comparação dos Processos}

Com os critérios de classificação e os formulários definidos e preenchidos de forma inicial, foi realizado o mapeamento dos modelos MPS e CMMI-DEV, comparando-se os componentes obrigatórios dos dois modelos, sempre partindo dos resultados esperados dos processos do modelo MPS, em comparação com as práticas específicas do modelo CMMI-DEV. Desta forma, nesta etapa foram preenchidas as demais colunas do formulário, tendo-se, assim, uma primeira versão do mapeamento. No caso do processo Gerência de Requisitos (GRE) foi adotado o primeiro modelo de formulário (Figura 8).

De forma semelhante, foi realizada a comparação dos dois modelos, utilizandose o segundo, terceiro e quarto modelos de formulário, quando todos os processos e áreas de processos, e respectivos componentes obrigatórios, foram analisados. No desenvolvimento do mapeamento foi considerada a representação por estágios do CMMIDEV, pois o MR-MPS não possui equivalente ao modelo contínuo do CMMI. Assim, não foram mapeados o objetivo genérico GG 1 e a prática genérica GP 1.1, bem como o atributo de processo AP 1 e o resultado do atributo de processo RAP 1 do modelo MPS. 


\begin{tabular}{|l|l|l|l|l|}
\hline \multicolumn{2}{|l|}{ Resultado Esperado do Processo } & Objetivo e Prática Específica & \multicolumn{1}{|c|}{ Classificação e Considerações } \\
\hline GRE 1 & $\begin{array}{l}\text { Os requisitos são entendi- } 1 \\
\text { dos, avaliados e aceitos } \\
\text { junto aos fornecedores de } \\
\text { requisitos, utilizando } \\
\text { critérios objetivos. }\end{array}$ & $\begin{array}{l}\text { Os requisitos são gerenciados e as inconsistências são identificadas } \\
\text { em relação aos planos de projeto e produtos de trabalho. }\end{array}$ \\
\hline $\begin{array}{l}\text { Trabalhar com } \\
\text { os provedores } \\
\text { de requisitos } \\
\text { para obter um } \\
\text { melhor enten- } \\
\text { dimento do } \\
\text { significado dos } \\
\text { requisitos. }\end{array}$ & $\begin{array}{l}\text { O MR-MPS exige que os requisitos sejam } \\
\text { entendidos, avaliados e aceitos juntos aos } \\
\text { fornecedores de requisitos, com adoção de } \\
\text { critérios objetivos. Embora não explícito na } \\
\text { descrição da prática, o CMMI-DEV também } \\
\text { exige o entendimento, avaliação e o aceite dos } \\
\text { requisitos. Porém, só há referência ao estabe- } \\
\text { lecimento de critérios objetivos nas subpráti- } \\
\text { cas, o que não constitui uma obrigatoriedade. }\end{array}$ \\
\hline
\end{tabular}

Figura 8 - Exemplo do Mapeamento do processo GRE

\subsection{Avaliação Através de Revisão por Pares}

Após a conclusão da comparação de cada processo do MR-MPS:2009 para a área de processo relacionada do CMMI-DEV, o mapeamento inicial foi objeto de revisão por pares, incluindo-se a avaliação dos resultados de atributos de processo do MR-MPS em relação às práticas genéricas do CMMI-DEV, até o nível $\mathrm{C}$ de maturidade, e dos resultados de atributos de processo dos níveis $\mathrm{A}$ e $\mathrm{B}$ de maturidade em relação às práticas genéricas e áreas de processo dos níveis 4 e 5.

A revisão por pares teve os seguintes objetivos: (i) avaliar se os critérios de classificação eram adequados; (ii) avaliar se a correspondência entre os processos e áreas de processos, entre os resultados esperados do processo e as práticas específicas e entre os atributos de processo e resultados do atributo de processo e os objetivos genéricos e as práticas genéricas, respectivamente dos modelos MPS e CMMI-DEV, estavam coerentes com a interpretação das definições; e (iii) avaliar se o conjunto de considerações permitia esclarecer a classificação atribuída na comparação entre os modelos.

A seleção dos revisores foi realizada a partir do grupo de implementadores e avaliadores do modelo MPS, em uma amostragem baseada em conveniência (disponibilidade para realizar a revisão) e a partir do critério de possuir conhecimento também no modelo CMMI-DEV. Assim, cada processo até o nível $\mathrm{C}$ de maturidade do modelo MPS foi avaliado por pelo menos dois revisores diferentes.

Para realizar a revisão por pares da alta maturidade - níveis B e A do modelo MPS e 4 e 5 do modelo CMMI-DEV - foram selecionados como potenciais revisores todos os avaliadores MPS habilitados a avaliar a alta maturidade, excluídos os orientadores da dissertação que originou esse artigo, mais os lead appraiser SCAMPI habilitados pelo SEI a avaliar a alta maturidade e que possuíam conhecimento do modelo MPS, por terem participado do Curso Oficial de Introdução ao MPS. Com este critério, foram selecionados cinco potenciais revisores, aos quais foram enviados os mapeamentos iniciais relacionados à alta maturidade. Destes, quatro revisores realizaram a revisão por pares e contribuíram para a versão atual do mapeamento.

Para apoiar a revisão por pares, uma planilha de apoio foi elaborada, tendo sido adaptada da planilha usualmente utilizada nas revisões por pares realizadas pela Equipe Técnica do Modelo MPS. Com isso, o mapeamento de cada processo foi encaminhado por e-mail aos pesquisadores descrevendo o contexto e o objetivo do trabalho, juntamente com a planilha. Utilizando esta planilha, os revisores podiam classificar cada comentário relacionado ao mapeamento em uma das seguintes categorias: TA (Técnico Alto), indicando que foi encontrado um problema em um item que, se não for alterado, comprometerá as considerações; TB (Técnico Baixo), indicando que foi encontrado um 
problema em um item que seria conveniente alterar; E (Editorial), indicando que foi encontrado um erro de português ou que o texto podia ser melhorado; Q (Questionamento), indicando que houve dúvidas quanto ao conteúdo das considerações; e G (Geral), indicando que o comentário é geral em relação às considerações (Figura 9).

\begin{tabular}{|c|c|c|l|l|}
\hline \hline ID & $\begin{array}{c}\text { Categoria } \\
\text { (TA, TB, E, } \\
\text { Q, G) }\end{array}$ & Item & Comentário com a justificativa & Novo Texto Proposto \\
\hline 5 & TA & GRI.4 & $\begin{array}{l}\text { A justificativa para o "Não equivamente" } \\
\text { não é válida, pois na descrição (última } \\
\text { frase do primeiro parágrafo) do SP 2.1 é } \\
\text { informado que "Risks are documented in } \\
\text { a concise statement that includes the } \\
\text { context, conditions, and consequences of } \\
\text { risk occurrence" }\end{array}$ & Há equivalência entre GRI.4 e SP 2.1 \\
\hline
\end{tabular}

Figura 9 - Fragmento dos comentários da planilha de revisão por pares

Após o recebimento das planilhas de avaliação de todos os processos, os resultados foram organizados para subsidiar a análise. Está análise do retorno da revisão por pares foi dividida em duas partes, sendo a primeira para os processos até os níveis $\mathrm{C}$ e 3 de maturidade dos modelos MPS e CMMI-DEV; e a segunda para a alta maturidade, envolvendo os níveis B e A do modelo MPS e 4 e 5 do modelo CMMI-DEV. Todos os resultados da revisão por pares são apresentados em (MELLO, 2011).

\section{Avaliação do Mapeamento na Indústria}

A avaliação do mapeamento foi realizada através de dois estudos de caso em situação real na indústria, com o objetivo de avaliar a corretude, a adequação dos critérios de comparação, o nível de detalhe, a utilidade das considerações e o desempenho dos avaliadores durante a avaliação. O planejamento definido para condução dos dois estudos de caso, contemplou os seguintes aspectos: propósito, questões específicas de pesquisa, contexto, período de realização, participantes, instrumentos de apoio e ameaças à sua validade. Mais detalhes podem ser vistos em (MELLO, 2011).

O primeiro estudo de caso foi realizado em uma organização de desenvolvimento de software da cidade do Rio de Janeiro/RJ, no segundo semestre de 2010, durante cinco dias consecutivos, e foi executado dentro do contexto de uma avaliação conjunta de processos MPS nível C e CMMI nível 3. Neste cenário, apenas o processo de Aquisição do modelo MPS e a área de processo Gerência de Acordo com Fornecedores do CMMI-DEV não foram avaliados. Isto ocorreu porque a organização não subcontrata produtos ou componentes para integrar o produto ou serviço entregue para o cliente, e declarou este processo/área de processo fora do escopo da avaliação. Seis avaliadores da equipe de avaliação participaram desse estudo de caso: um avaliador líder experiente MPS, dois avaliadores adjuntos MPS, um lead appraiser CMMI e dois representantes da organização avaliada. Três miniequipes foram constituídas e formadas por dois integrantes da equipe de avaliação. Cada miniequipe foi responsável pela avaliação de um conjunto de processos/áreas de processo.

Por ser uma avaliação conjunta, os avaliadores deveriam possuir experiência prévia nos dois modelos. No caso dos representantes da unidade organizacional, houve capacitação anterior através do curso oficial de Introdução ao MPS e no curso oficial de Introdução ao CMMI-DEV. Além dos seis participantes, o pesquisador responsável pelo mapeamento foi autorizado pelo patrocinador da avaliação a participar como observador. Em atendimento aos métodos de avaliação SCAMPI (SEI, 2006c) e MA-MPS 
(SOFTEX, 2009b), esta participação ocorreu apenas na avaliação inicial e foi restrita às observações, dúvidas e anotações sobre o mapeamento e sobre o formulário de resposta.

O segundo estudo de caso foi realizado em uma organização de desenvolvimento de software em Fortaleza/CE, no segundo semestre de 2010, em cinco dias consecutivos. Foi executado durante uma avaliação conjunta MPS nível E e CMMI nível 2 e, também, apenas na etapa de avaliação inicial. Com exceção do processo de Aquisição do modelo MPS e da área de processo Gerência de Acordo com Fornecedores do CMMI-DEV, excluídos por terem sido declarados fora de escopo, os demais processos foram avaliados. Quatro avaliadores da equipe de avaliação participaram do segundo estudo de caso: um avaliador líder experiente MPS, um avaliador adjunto MPS, um lead appraiser CMMI e um representante da organização avaliada. Duas miniequipes foram formadas, cada uma com dois avaliadores, todos com experiência nos dois modelos.

Para apoiar a execução dos estudos de caso no contexto da avaliação conjunta MPS/CMMI, dois instrumentos foram definidos: um Instrumento de Apoio à Avaliação Conjunta de Processos e um Formulário de Avaliação do Mapeamento.

O Instrumento de Apoio à Avaliação Conjunta de Processos (IACP) baseado nos modelos MPS e CMMI-DEV, foi elaborado com o objetivo de apoiar a condução das avaliações conjuntas MPS/CMMI. O IACP foi definido a partir da planilha oficial de avaliação do modelo MPS, adaptada para inclusão do componente correspondente do modelo CMMI-DEV, da classificação definida no mapeamento e das considerações relacionadas. Assim, foi mantida a mesma estrutura da planilha oficial MPS, com introdução de informações resultantes do mapeamento realizado. O IACP foi composto por vinte e uma planilhas, sendo uma para caracterização dos objetivos específicos e genéricos do CMMI, uma para as instruções de preenchimento e outras dezenove planilhas referentes aos processos, que foram preparadas e tiveram seu conteúdo atualizado a partir do mapeamento. Um exemplo de planilha para avaliações conjuntas MRMPS/CMMI-DEV pode ser visto na Figura 10. Os itens destacados evidenciam as diferenças para a planilha de avaliações exclusiva do MR-MPS.

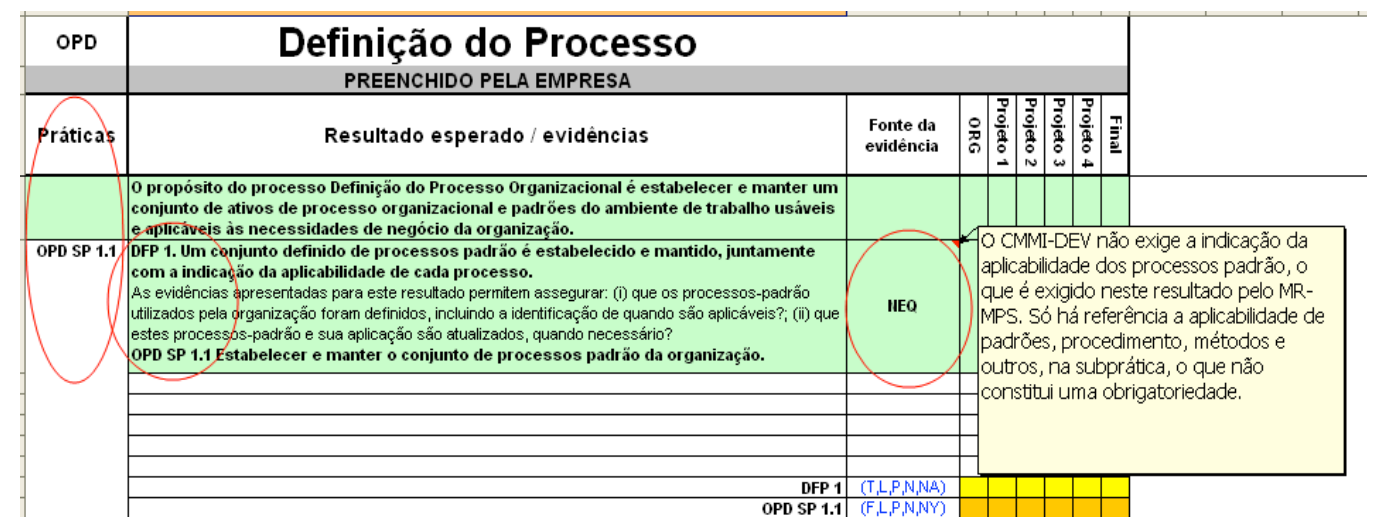

Figura 10 - Exemplo de Planilha de Avaliação Conjunta do MR-MPS/CMMI-DEV

O Formulário de Avaliação do Mapeamento foi elaborado para ser preenchido pela equipe de avaliação. Com cinco questões, o formulário visou capturar informações referentes a corretude do mapeamento, nível de detalhe, adequação dos critérios de classificação, utilidade das considerações e desempenho das equipes de avaliação.

Após a realização dos estudos, os resultados foram consolidados a partir da análise dos 23 formulários de avaliação do mapeamento preenchidos, por questão avaliada, 
sendo 15 do primeiro e 8 do segundo estudo de caso. Como pode ser observado, todos os participantes consideraram que os critérios de classificação adotados na comparação foram adequados e que o desempenho das equipes na avaliação conjunta foi facilitado com o mapeamento (Figura 11).

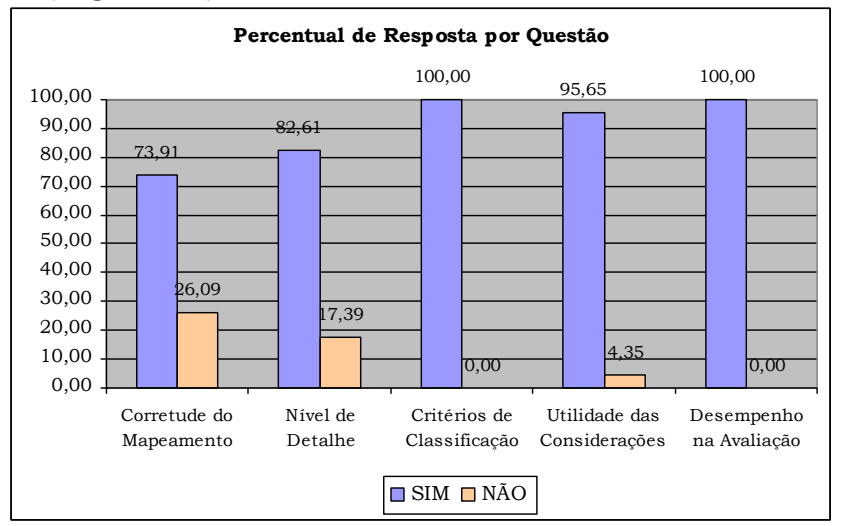

Figura 11 - Percentual de Resposta por Questão

Com relação à utilidade das considerações para esclarecer a classificação atribuída na comparação entre os modelos, $95,65 \%$ dos formulários respondidos indicaram que elas foram úteis, enquanto que $82,61 \%$ consideraram que o nível de detalhe do mapeamento foi suficiente e $73,91 \%$ que o mapeamento entre os modelos estava correto. Note que esse último número consolida todas as discordâncias em relação ao mapeamento apresentando, mesmo que os pontos levantados nos formulários apresentasse uma pequena discordância. Dessa forma, após análise das observações registradas, considerou-se o resultado satisfatório.

Das cinco miniequipes formadas nos dois estudos de caso, apenas a miniequipe 1 do segundo estudo de caso não relatou observações nos formulários de avaliação. Com isso, de um total de 21 observações feitas nos dois estudos de caso, 17 observações foram do primeiro e 4 foram do segundo estudo de caso. Com relação aos resultados específicos por processo, do total de 15 processos avaliados nos dois estudos de caso, 7 processos não tiveram observação para melhoria, a saber: GRE, GCO, GQA, MED, DFP, DRE e GDE. Para os outros 8 processos, foram registradas 20 observações de correção ou evolução, além de uma observação relacionada aos RAPs, em comparação aos objetivos e práticas genéricos. As 21 observações foram analisadas e contribuíram para aprimoramento do mapeamento realizando, resultando em sua versão atual.

\section{Conclusão}

Este trabalho apresentou uma metodologia de pesquisa elaborada para mapeamento dos modelos MPS e CMMI-DEV, realizado com o objetivo de auxiliar as organizações nas iniciativas de melhoria de processos de software multi-modelos, seja no âmbito das implementações ou das avaliações de processos. As iniciativas e trabalhos relacionados a melhoria de processos de software multi-modelos foram apresentados, envolvendo tanto relatos em organizações quanto as pesquisas relacionadas a mapeamento, integração e harmonização de normas e modelos de referência.

Como principais contribuições desse trabalho destacam-se (i) o subsídio para elaboração do Guia de Implementação do MR-MPS Parte 11 (SOFTEX, 2011), contendo orientações para a implementação e avaliação do MPS em conjunto com o CMMIDEV e (ii) a elaboração da planilha de avaliação dos modelos MPS e CMMI, hoje dis- 
ponível para utilização pela equipes de avaliação nos processos de avaliações conjuntas e complementares. Essa planilha já foi utilizada outras vezes além dos momentos descritos nos estudos realizados, sendo a mais recente em avaliação complementar nível $\mathrm{F}$ do MR-MPS e nível 2 do CMMI2 em dezembro de 2011 em uma organização em João Pessoa. Um trabalho futuro é a revisão do mapeamento e da planilha para as versões 2011 do MR-MPS e da versão 1.3 do CMMI-DEV.

\section{Agradecimentos}

Os autores agradecem à FAPERJ pelo auxílio financeiro (projeto E-26/110.399/2011).

\section{Referências}

ARAÚJO, R., CAPPELLI, C., GOMES, A., PEREIRA, M., IENDRIKE, H.S., IELPO, D., TOVAR, J.A, 2008., "A Definição de Processos de Software sob o ponto de vista da Gestão de Processos de Negócio”. VI Simpósio Internacional de Melhoria de Processos de Software, São Paulo.

BALDASSARRE, M.T., CAIVANO, D., PINO, F.J., PIATTINI, M., VISAGGIO, G., 2010., "A Strategy for Painless Harmonization of Quality Standards: A Real Case". PROFES 2010, LNCS 6156, pp 395-408.

BASILI, V., ROMBACH, H., 1988, "The Tame Project: Towards ImprovementOriented Software Environments". IEEE Transactions on Software Engineering, v. 14, n. 6, pp. 758-773.

BECKER, C.A, 2007, Prikladnicki, R., Galarraga, O., "Cooperativa MPS.BR - Relato de experiências, lições aprendidas, melhores práticas e dificuldades da II e IOGE SOFTSUL do RS". II Workshop de IOGES (W5 - MPS.BR), em Belo Horizonte, nos dias 28 e 29 de novembro de 2007. Disponível em: http://www.softex.br/mpsbr.

CHANWOO, Y., Yoon, J., LEE, B., CHONGWON, L., JINYOUNG, L., WU, C., 2004; Seoul National University; "AN Integrated Model of ISO 9001:2000 and CMMI for ISO Registered Organizations"; 11th APSEC.

FERREIRA, A.I.F., SANTOS, G., CERQUEIRA, R., SANTOS, G.; MONTONI, M.; BARRETO, A.S.; ROCHA, A.R, 2006, "ISO 9001:2000, MPS.BR Nível F e CMMI Nível 3: Uma Estratégia de Melhoria de Processos na BL Informática". Simpósio Brasileiro de Qualidade de Software 2006, SBQS 2006.

FERREIRA, A.L., MACHADO, R.J., PAULK, M.C., 2010, "Size and Complexity Attributes for Multimodel Improvement Framework Taxonomy", 36th EUROMICRO Conference on Software Engineering and Advanced Applications, 2010.

ISO, 2008, "ISO 9001:2008 - Quality management systems - Requirement", The International Organization for the Standardization and the International Electrotechnical Commission.

KIRWAN, P.; SIVIY, J., 2008b, "Process improvement in multi-model environments (PrIME)". Disponível em http://www.sei.cmu.edu/library/abstracts/ webinars/18jul2008.cfm.

KITCHENHAM, B., 2008, "Procedures for Performing Systematic Reviews, Technical Report", Departament of Computer Science Keele University, Keele.

LEPASAAR, M., MÄKINEN, T., 2002. "Integrating software process assessment models using a process meta model". 2002 IEEE International Engineering Management Conference 1, pp. 224-229.

MAFRA, S. N., TRAVASSOS, G. H., 2006, "Estudos Primários e Secundários apoiando a busca por Evidência em Engenharia de Software". Relatório Técnico ES- 
687/06, PESC/COPPE/UFRJ, Rio de Janeiro, Brasil.

MELLO, M., 2011, "Melhoria de Processos de Software Multi-Modelos Baseada nos Modelos MPS e CMMI-DEV”. Dissertação de Mestrado, COPPE/UFRJ, Rio de Janeiro, RJ, Brasil.

MELLO, M., ROCHA, A. R. C., 2009. “Gestão Integrada da Melhoria de Processos em Organizações de Software”. V Workshop Anual do MPS. WAMPS 2009 - Campinas/SP.

MUTAFELIJA, B; STROMBERG, H., 2009, "Process Improvement with CMMI v 1.2 and ISO Standards", CRC Press.

NUNES, E. D., SILVA, R., ROCHA, A.R, NATALI, A.C., SANTOS, G. , 2005, "Uma Abordagem para Implantação de Processos de Software com ISO 9001 e CMMI" SBQS, 2005.

PINO, F.J., BALDASSARRE, M.T., PIATTINI, M., VISAGGIO, G., 2010, "Harmonizing maturity levels from CMMI-DEV and ISO/IEC 15504". Journal of Software Maintenance and Evolution 22 (4), pp. 279-296.

RESENDE, D.K., G.,J.B., P., N., G., C.A., J., E. N. V, F., A.C., KRUEL, F., BATISTA, P.R., NETO, O.C.T., CAVALCANTI, W. GODINHO, H., MONTONI, M., NUNES, E., BARRETO, A., ROCHA, A.R., 2009., "Implementação do MPS.BR Nível F e CMMI-DEV Nível 2 na Red \& White IT Solutions". WAMPS 2009.

ROUT, T.P; TUFFLEY, A., 2007. "Harmonizing ISO/IEC 15504 AND CMMI". SPI; 2007;12: 361-371, InterScience

SEI, 2006a, SOFTWARE ENGENEERING INSTITUTE, "CMMI for Development, Version 1.2”, CMMI-DEV v1.2, CMU/SEI 2006-TR-008, Technical Report, Software Engineering Institute, August 2006a.

SILVA FILHO, R. C., 2006, "Uma Abordagem para Avaliação de Propostas de Melhoria em Processos de Software”. Dissertação de M.Sc., COPPE/UFRJ, Rio de Janeiro, RJ, Brasil.

SOFTEX, 2009a. "MPS.BR: Melhoria de Processo do Software Brasileiro, Guia Geral (v. 2009)".

SOFTEX, 2011, "MPS.BR - Melhoria de Processo do Software Brasileiro - Guia de Implementação - Parte 11: Orientações para a implementação e avaliação do Modelo de Referência MR-MPS:2009 em conjunto com o CMMI-DEV v1.2”. Disponível em: http:www.softex.br/mpsbr

SOUZA, C., ROCHA, A.R., RUBINSTEIN, A., MAGALHAES, A.L., KATSURAYAMA, A., DUQUE, A, BARBIERI, C., CERDEIRAL, C., TEIXEIRA, L., PAIVA, N. S., BARROS, L. , 2009, “Avaliação Conjunta CMMI Nível 3 e MPS Nível C: Lições Aprendidas e Recomendações". WAMPS 2009.

STAPLES, M., NIAZI, M., JEFFERY, R., et al., 2007, "An exploratory study of why organizations do not adopt CMMI". Journal Syst. Soft., v. 80, n. 6, pp. 883-895.

THIRY, M., HAUCK, J. C., WANGENHEIM, C. G. V., SOUZA, R. H., 2008a, "Process Reference Guides - Support for Improvement Software Processes in Alignment with Reference Models and Standards". EuroSPI, CCIS 16, pp 70-81.

THIRY, M.; WANGENHEIM, C.; ZOUCAS, A.; TRISTÃO, L.; 2008b. "FAPS: Ferramenta para apoiar Avaliações Integradas de Processos de Software". IV Workshop de Implementadores (W2-MPS.BR), Campinas, out/2008. Em www.softex.br

WÖHLIN, C., RUNESON, P., HÖST, M., OHLSSON, M., REGNELL, B., WESSLÉN, A., 2000, "Experimentation in Software Engineering: An Introduction", The Kluwer International Series in Soft. Eng., Norwell, USA, Kluwer Academic Publishers. 